PROCEEDINGS OF THE

AMERICAN MATHEMATICAL SOCIETY

Volume 132, Number 3, Pages 807-813

S 0002-9939(03)07170-3

Article electronically published on July 7, 2003

\title{
ON A LIOUVILLE-TYPE THEOREM AND THE FUJITA BLOW-UP PHENOMENON
}

\author{
A. G. KARTSATOS AND V. V. KURTA
}

(Communicated by David S. Tartakoff)

\begin{abstract}
The main purpose of this paper is to obtain the well-known results of H. Fujita and K. Hayakawa on the nonexistence of nontrivial nonnegative global solutions for the Cauchy problem for the equation

$(*)$

$$
u_{t}=\Delta u+|u|^{q-1} u
$$

with $q \in\left(1,1+\frac{2}{n}\right]$ on the half-space $\mathbb{S}:=(0,+\infty) \times \mathbb{R}^{n}, n \geq 1$, as a consequence of a new Liouville theorem of elliptic type for solutions of $(*)$ on $\mathbb{S}$. This new result is in turn a consequence of other new phenomena established for nonlinear evolution problems. In particular, we prove that the inequality

$$
|u|_{t} \geq \Delta u+|u|^{q},
$$

has no nontrivial solutions on $\mathbb{S}$ when $q \in\left(1,1+\frac{2}{n}\right]$. We also show that the inequality

$$
u_{t} \geq \Delta u+|u|^{q-1} u
$$

has no nontrivial nonnegative solutions for $q \in\left(1,1+\frac{2}{n}\right]$, and it has no solutions on $\mathbb{S}$ bounded below by a positive constant for $q>1$.
\end{abstract}

\section{INTRODUCTION AND PRELIMINARIES}

We obtain the well-known results of H. Fujita and K. Hayakawa ([1, 2]) on the nonexistence of nontrivial nonnegative global solutions for the Cauchy problem for the equation

$$
u_{t}=\Delta u+|u|^{q-1} u
$$

with any fixed $q \in\left(1,1+\frac{2}{n}\right]$ on the half-space $\mathbb{S}:=(0,+\infty) \times \mathbb{R}^{n}, n \geq 1$, as a consequence of a new result on the nonexistence of nontrivial nonnegative "entire" (defined on the whole half-space) solutions for (1) without taking their traces on the hyperplane $t=0$ into account. This new result, which we naturally call a Liouville theorem of elliptic type, is especially interesting in connection with weak solutions on $\mathbb{S}$, which have no "good" traces on $t=0$.

Actually, we obtain a Liouville theorem of elliptic type for nonnegative solutions of equation (1) on the half-space $\mathbb{S}$ as a consequence of another new phenomenon established for nonlinear evolution problems. Namely, we prove that for any fixed

Received by the editors October 30, 2002.

2000 Mathematics Subject Classification. Primary 35K55, 35R45, 35B40.

Key words and phrases. Cauchy problem, entire solution, blow-up, Fujita phenomenon, global solution, Liouville theorem. 
$q \in\left(1,1+\frac{2}{n}\right]$ the inequality

$$
|u|_{t} \geq \Delta u+|u|^{q}
$$

has no nontrivial solutions on $\mathbb{S}$. As a simple consequence of this result we obtain that for any fixed $q \in\left(1,1+\frac{2}{n}\right]$ the inequality

$$
u_{t} \geq \Delta u+|u|^{q-1} u
$$

has no nontrivial nonnegative solutions on $\mathbb{S}$. We also prove that for $q>1$ inequality (3) has no solutions on $\mathbb{S}$ bounded below by a positive constant.

The approach developed herein is directly applicable to obtaining similar blowup results for wide classes of equations, inequalities and systems of equations and inequalities with linear and nonlinear differential operators, considered on the halfspace $\mathbb{S}$ as well as on Riemannian manifolds; in particular, it is applicable to obtaining similar Liouville-type results for inhomogeneous analogues of (1)-(3), for example, in the framework of 3 .

\section{The RESUlts}

In what follows, $q \geq 1$ and the symbol $\stackrel{\circ}{C}^{\infty}(\mathbb{S})$ denotes the space of all $C^{\infty}$ _ functions $f: \mathbb{S} \rightarrow \mathbb{R}$ with compact support.

Definition 1. By a solution of (1) on $\mathbb{S}$ we understand a function $u(t, x)$ which belongs to the space $L_{q, l o c}(\mathbb{S})$ and satisfies the integral equation

$$
\int_{\mathbb{S}}\left[-u \varphi_{t}-u \Delta \varphi\right] d t d x=\int_{\mathbb{S}} u|u|^{q-1} \varphi d t d x
$$

for any function $\varphi \in \stackrel{\circ}{C}^{\infty}(\mathbb{S})$.

Theorem 1. Let $1<q \leq 1+\frac{2}{n}$, and let $u(t, x)$ be a nonnegative solution of (1) on $\mathbb{S}$. Then $u(t, x)=0$ a.e. on $\mathbb{S}$.

Definition 2. By a solution of $(2)$ on $\mathbb{S}$ we understand a function $u(t, x)$ which belongs to the space $L_{q, l o c}(\mathbb{S})$ and satisfies the integral inequality

$$
\int_{\mathbb{S}}\left[-|u| \varphi_{t}-u \Delta \varphi\right] d t d x \geq \int_{\mathbb{S}}|u|^{q} \varphi d t d x
$$

for any nonnegative function $\varphi \in \stackrel{\circ}{C}^{\infty}(\mathbb{S})$.

Theorem 2. Let $1<q \leq 1+\frac{2}{n}$, and let $u(t, x)$ be a solution of (2) on $\mathbb{S}$. Then $u(t, x)=0$ a.e. on $\mathbb{S}$.

In addition to Theorem 2, we obtain below (Proposition 1) an a priori estimate for solutions of (2), which is of independent interest and is needed in the proof of Theorem 2.

In what follows, $P(r):=\left\{(t, x) \in \mathbb{S}: t+|x|^{2}<r\right\}$ for any fixed $r>0$.

Proposition 1. Let $q>1$, and let $u(t, x)$ be a solution of (2) on $\mathbb{S}$. Then there exists a constant $c>0$ such that the inequality

$$
c R^{\frac{n+2}{2}-\frac{q}{q-1}} \geq \int_{P(R)}|u|^{q} d t d x
$$

holds for any $R>0$. 
Definition 3. By a solution of (3) on $\mathbb{S}$ we understand a function $u(t, x)$ which belongs to the space $L_{q, l o c}(\mathbb{S})$ and satisfies the integral inequality

$$
\int_{\mathbb{S}}\left[-u \varphi_{t}-u \Delta \varphi\right] d t d x \geq \int_{\mathbb{S}} u|u|^{q-1} \varphi d t d x
$$

for any nonnegative function $\varphi \in \stackrel{\circ}{C}^{\infty}(\mathbb{S})$.

Theorem 3. Let $1<q \leq 1+\frac{2}{n}$, and let $u(t, x)$ be a nonnegative solution of (3) on $\mathbb{S}$. Then $u(t, x)=0$ a.e. on $\mathbb{S}$.

Remark 1. Since the nonnegative solutions of (1) and (3) are solutions of (2), Theorems 1 and 3 are simple consequences of Theorem 2. In addition, a priori estimates for nonnegative solutions of (1) and (3) hold, and are similar to the estimate of Proposition 1.

Remark 2. It is clear that in the case $q=1$ and $q \in(-\infty, 1]$ the function $u(t, x):=e^{t}$ is a positive classical solution of equation (1) and inequalities (2) and (3), respectively, on $\mathbb{S}$. If $q>1+\frac{2}{n}$, then, by the well-known results on the existence of nontrivial nonnegative global solutions of the Cauchy problem for equation (1) (see [1] or, e.g., [4, 6]), it is clear that there exist nontrivial nonnegative solutions of $(1),(2)$ and $(3)$ on $\mathbb{S}$.

However, the nonexistence of nonnegative solutions of (1) and (3) on $\mathbb{S}$, bounded below by a positive constant, is covered by Theorem 4 below. We need the following definition.

Definition 4. A function $u \in L_{1, l o c}(\mathbb{S})$ is said to be bounded below by a positive constant on $\mathbb{S}$ if there exists a constant $K=K(u)>0$ such that $u(t, x) \geq K$ a.e. on $\mathbb{S}$.

Theorem 4. Let $q>1$. Then there exist no solutions of (1) and (3) on $\mathbb{S}$ bounded below by a positive constant.

Remark 3 . Since Theorem 1 is a simple consequence of Theorem 3, and since we impose no conditions on the behavior of the solutions of (1) and (3) on the hyperplane $t=0$, the nonexistence of nontrivial nonnegative global solutions for the Cauchy problem with arbitrary initial data for (1) and (3) with $q \in\left(1, \frac{2}{n}\right]$, in particular, the famous blow-up results of Fujita and Hayakawa ([1], [2]), are special cases of Theorem 3. Similar results hold for solutions of inequality (2), namely, the nonexistence of nontrivial global solutions for the Cauchy problem with arbitrary initial data for (2) with $q \in\left(1, \frac{2}{n}\right]$ is a special case of Theorem 2. Also, the nonexistence of nontrivial global solutions, bounded below by a positive constant, for the Cauchy problem with arbitrary initial data for (1) and (3) with any fixed $q>1$ is a special case of Theorem 4. For a survey of the literature on blow-up results for the Cauchy problem we refer to [4]-[6].

\section{The PROOFS}

In what follows, a "smooth" function is a $C^{\infty}$-function.

Proof of Theorem 2. Let $q>1$ and let $u(t, x)$ be a solution of $(2)$ on $\mathbb{S}$. Let $0<\tau<$ $+\infty$ and $0<r<R<+\infty$. Let $\eta:[0,+\infty) \rightarrow[0,1]$ be a smooth function which has the nonnegative derivative $\eta^{\prime}$ and equals 0 on the interval $[0, \tau]$ and 1 on the 
interval $[2 \tau,+\infty)$. Let $\zeta:[0,+\infty) \times \mathbb{R}^{n} \rightarrow[0,1]$ be a smooth function which equals 1 on $\overline{P(r)}$ and 0 on $\left\{[0,+\infty) \times \mathbb{R}^{n}\right\} \backslash \overline{P(R)}$. Substituting $\varphi(t, x)=\zeta^{s}(t, x) \eta^{2}(t)$ as a test function in $\left(2^{\prime}\right)$, where the positive constant $s \geq 2$ will be chosen below, we obtain

$$
\begin{aligned}
(5)-s \int_{P(R)}|u| \zeta_{t} \zeta^{s-1} \eta^{2} d t d x-2 \int_{P(R)}|u| \zeta^{s} \eta^{\prime} \eta d t d x & \\
& -\int_{P(R)} u \eta^{2} \Delta \zeta^{s} d t d x \geq \int_{P(R)}|u|^{q} \zeta^{s} \eta^{2} d t d x .
\end{aligned}
$$

Since $\eta^{\prime} \geq 0$ for all $t>0$, the second integral on the left-hand side of (5) is nonnegative. Therefore,

$$
s \int_{P(R)}|u|\left|\zeta_{t}\right| \zeta^{s-1} \eta^{2} d t d x+\int_{P(R)}|u|\left|\Delta \zeta^{s}\right| \eta^{2} d t d x \geq \int_{P(R)}|u|^{q} \zeta^{s} \eta^{2} d t d x .
$$

Since

$$
\Delta \zeta^{s}=s \zeta^{s-1} \Delta \zeta+s(s-1) \zeta^{s-2}\left|\nabla_{x} \zeta\right|^{2},
$$

it follows easily from (6) that

$$
\begin{aligned}
s \int_{P(R)}|u|\left|\zeta_{t}\right| \zeta^{s-1} \eta^{2} d t d x+s \int_{P(R)}|u||\Delta \zeta| \zeta^{s-1} \eta^{2} d t d x & \\
& +s(s-1) \int_{P(R)}|u|\left|\nabla_{x} \zeta\right|^{2} \zeta^{s-2} \eta^{2} d t d x \geq \int_{P(R)}|u|^{q} \zeta^{s} \eta^{2} d t d x .
\end{aligned}
$$

Estimating all the integrals on the left-hand side of (7) by Hölder's inequality, we arrive at

$$
\begin{aligned}
& \left(\int_{P(R) \backslash P(r)}|u|^{q} \zeta^{s} \eta^{2} d t d x\right)^{\frac{1}{q}}\left(\int_{P(R)}\left|\zeta_{t}\right|^{\frac{q}{q-1}} \zeta^{s-\frac{q}{q-1}} \eta^{2} d t d x\right)^{\frac{q-1}{q}} \\
& +\left(\int_{P(R) \backslash P(r)}|u|^{q} \zeta^{s} \eta^{2} d t d x\right)^{\frac{1}{q}}\left(\int_{P(R)}|\Delta \zeta|^{\frac{q}{q-1}} \zeta^{s-\frac{q}{q-1}} \eta^{2} d t d x\right)^{\frac{q-1}{q}} \\
& +\left(\int_{P(R) \backslash P(r)}|u|^{q} \zeta^{s} \eta^{2} d t d x\right)^{\frac{1}{q}}\left(\int_{P(R)}\left|\nabla_{x} \zeta\right|^{\frac{2 q}{q-1}} \zeta^{s-\frac{2 q}{q-1}} \eta^{2} d t d x\right)^{\frac{q-1}{q}} \\
& \geq c_{1} \int_{P(R)}|u|^{q} \zeta^{s} \eta^{2} d t d x .
\end{aligned}
$$

In what follows, we use the symbols $c_{i}, i=1,2, \ldots$, to denote constants depending on $n, q$, and $s$, but not on $\tau, r$, or $R$.

Now, we choose in (8) the function $\zeta(t, x):=\psi\left(\left(t+|x|^{2}\right) / R\right)$, where $\psi:[0,+\infty) \rightarrow$ $[0,1]$ is a smooth function such that $\psi=1$ on $[0,1 / 2]$ and $\psi=0$ on $[1,+\infty)$. Since, 
for arbitrary $R=2 r>0$ and $(t, x) \in \mathbb{S}$, the volume of $P(R)$ is less than or equal to $c_{2} R^{\frac{n+2}{2}}$ and $\psi$ can be chosen such that

$$
\left|\zeta_{t}\right| \leq c_{2} R^{-1}, \quad|\Delta \zeta| \leq c_{2} R^{-1}, \quad\left|\nabla_{x} \zeta\right|^{2} \leq c_{2} R^{-1}
$$

inequality (8) yields, for any fixed $s \geq \frac{2 q}{q-1}$,

$$
\left(\int_{P(R) \backslash P(r)}|u|^{q} \zeta^{s} \eta^{2} d t d x\right)^{\frac{1}{q}}\left(R^{\frac{n+2}{2}-\frac{q}{q-1}}\right)^{\frac{q-1}{q}} \geq c_{3} \int_{P(R)}|u|^{q} \zeta^{s} \eta^{2} d t d x .
$$

Furthermore, since

$$
\frac{n+2}{2}-\frac{q}{q-1} \leq 0 \quad \text { for } \quad 1<q \leq \frac{n+2}{n},
$$

it follows from (9) that $\int_{\mathbb{S}}|u|^{q} \eta^{2} d t d x$ is bounded. Thus, by monotonicity,

$$
\int_{P\left(2 r_{k}\right) \backslash P\left(r_{k}\right)}|u|^{q} \eta^{2} d t d x \rightarrow 0
$$

for any sequence $r_{k} \rightarrow+\infty$, which, combined with (9), gives

$$
\int_{P\left(r_{k}\right)}|u|^{q} \eta^{2} d t d x \rightarrow 0
$$

as $r_{k} \rightarrow+\infty$. This in turn implies $\int_{\mathbb{S}}|u|^{q} \eta^{2} d t d x=0$. Using the fact that the function $\eta(t)$ equals 1 on the interval $[2 \tau,+\infty)$ and $\tau>0$ is arbitrary, we obtain $u(t, x)=0$ a.e. on $\mathbb{S}$.

Proof of Proposition 1. Here, we use notation from the proof of Theorem 2 in order to prove a priori estimate (4) for solutions of (2). This estimate follows directly from inequality (9). Indeed, given $R=2 r>0$, inequality (9) implies, for a certain $c_{4}>0$,

$$
c_{4} R^{\frac{n+2}{2}-\frac{q}{q-1}} \geq \int_{P(R / 2)}|u|^{q} \eta^{2} d t d x
$$

where $\eta:[0,+\infty) \rightarrow[0,1]$ is a smooth function which equals 1 on the interval $[2 \tau,+\infty)$. Passing to the limit in (12) as $\tau \rightarrow 0$, we obtain inequality (4).

Proof of Theorem 4. We show our claim via contradiction. Let $q>1$ and let there exist a solution $u(t, x)$ of $(3)$ on $\mathbb{S}$ bounded below by a certain positive constant. As above, let $0<\tau<+\infty, 0<r<R<+\infty$. Let $\eta:[0,+\infty) \rightarrow[0,1]$ be a smooth function which has nonnegative derivative $\eta^{\prime}$, and equals 0 on the interval $[0, \tau]$ and 1 on the interval $[2 \tau,+\infty)$. Let $\zeta:[0,+\infty) \times \mathbb{R}^{n} \rightarrow[0,1]$ be a smooth function which equals 1 on $\overline{P(r)}$ and 0 on $\left\{[0,+\infty) \times \mathbb{R}^{n}\right\} \backslash \overline{P(R)}$. Substituting $\varphi(t, x)=\zeta^{s}(t, x) \eta^{2}(t)$ as a test function in $\left(3^{\prime}\right)$, where the positive constant $s \geq 2$ 
will be chosen below, we obtain

$$
\begin{aligned}
-s \int_{P(R)} u \zeta_{t} \zeta^{s-1} \eta^{2} d t d x-2 \int_{P(R)} u \zeta^{s} \eta^{\prime} \eta d t d x & \\
& -\int_{P(R)} u \eta^{2} \Delta \zeta^{s} d t d x \geq \int_{P(R)} u^{q-1} u \zeta^{s} \eta^{2} d t d x .
\end{aligned}
$$

Since $\eta^{\prime} \geq 0$ for all $t>0$, the second integral on the left-hand side of (13) is nonnegative. Therefore, (13) yields

$$
s \int_{P(R)} u\left|\zeta_{t}\right| \zeta^{s-1} \eta^{2} d t d x+\int_{P(R)} u\left|\Delta \zeta^{s}\right| \eta^{2} d t d x \geq \int_{P(R)} u^{q} \zeta^{s} \eta^{2} d t d x .
$$

Since for a nonnegative solution $u$ we have $u=|u|$, we may repeat the proof of Theorem 2 word for word from (6) to (9). As a result we obtain the inequality

$$
\left(\int_{P(R) \backslash P(r)} u^{q} \zeta^{s} \eta^{2} d t d x\right)^{\frac{1}{q}}\left(R^{\frac{n+2}{2}-\frac{q}{q-1}}\right)^{\frac{q-1}{q}} \geq c_{5} \int_{P(R)} u^{q} \zeta^{s} \eta^{2} d t d x .
$$

As above, we use the symbols $c_{i}, i=1,2, \ldots$, to denote constants depending on $n, q$, and $s$, but not on $\tau, r$, or $R$. It follows from (15) that

$$
c_{6} R^{\frac{n+2}{2}-\frac{q}{q-1}} \geq \int_{P(R / 2)} u^{q} \eta^{2} d t d x .
$$

Passing to the limit in (16) as $\tau \rightarrow 0$, we have

$$
c_{6} R^{\frac{n+2}{2}-\frac{q}{q-1}} \geq \int_{P(R / 2)} u^{q} d t d x .
$$

Since, by the assumption of Theorem $4, u(t, x)$ is bounded below by a positive constant and since the volume of $P(R / 2)$ is greater than or equal to $c_{7} R^{\frac{n+2}{2}}$, inequality (17) yields

$$
c_{8} R^{\frac{n+2}{2}-\frac{q}{q-1}} \geq R^{\frac{n+2}{2}}
$$

for any fixed $q>1$ and any $R>0$. Thus, for sufficiently large $R$, we have the contradiction to our assumption about the existence of solutions for (3) on $\mathbb{S}$ bounded below by a positive constant.

\section{REFERENCES}

1. H. Fujita, On the blowing up of solutions to the Cauchy problem for $u_{t}=\Delta u+u^{1+\alpha}$, J. Fac. Sci. Univ. Tokyo, Sect. 1A Math. 13 (1966), 109-124. MR 35:5761

2. K. Hayakawa, On nonexistence of global solutions of some semilinear parabolic differential equations, Proc. Japan Acad. Ser. A 49 (1973), 503-505. MR 49:3333

3. R. Pinsky, Finite time blow-up for the inhomogeneous equation $u_{t}=\Delta u+a(x) u^{p}+\lambda \varphi$ in $\mathbb{R}^{d}$, Proc. Amer. Math. Soc. 127 (1999), 3319-3327. MR 2000b:35116

4. H. A. Levine, The role of critical exponents in blowup theorems, SIAM Rev. 32 (1990), 262288. MR 91j:35135

5. A. A. Samarskii, V. A. Galaktionov, S. P. Kurdyumov and A. P. Mikhailov, Blow-up in Quasilinear Parabolic Equations, Walter de Gruyter, Berlin, 1995, 535 pp. MR 96b:35003 
6. K. Deng and H. A. Levine, The role of critical exponents in blow-up theorems: The sequel, J. Math. Anal. Appl. 243 (2000), 85-126. MR 2001b:35031

Department of Mathematics, University of South Florida, Tampa, Florida 33620-5700

E-mail address: hermes@math.usf.edu

Mathematical Reviews, 416 Fourth Street, P.O. Box 8604, Ann Arbor, Michigan 48107-8604

E-mail address: vvk@ams.org 